\title{
A SOFIA Survey of [C ii] in the galaxy M51 I. [C ii] as a tracer of Star Formation
}

DOI:

10.3847/2041-8213/aaf1ad

\section{Document Version}

Accepted author manuscript

Link to publication record in Manchester Research Explorer

\section{Citation for published version (APA):}

Pineda, J., Fischer, C., Kapala, M., Stutzki, J., Buchbender, C., Goldsmith, P., Ziebart, M., Glover, S. C. O., Klessen, R. S., Koda, J., Kramer, C., Mookerjea, B., Sandstrom, K., Scoville, N., \& Smith, R. (2018). A SOFIA Survey of [C ii] in the galaxy M51 I. [C ii] as a tracer of Star Formation. The Astrophysical Journal Letters. https://doi.org/10.3847/2041-8213/aaf1ad

\section{Published in:}

The Astrophysical Journal Letters

\section{Citing this paper}

Please note that where the full-text provided on Manchester Research Explorer is the Author Accepted Manuscript or Proof version this may differ from the final Published version. If citing, it is advised that you check and use the publisher's definitive version.

\section{General rights}

Copyright and moral rights for the publications made accessible in the Research Explorer are retained by the authors and/or other copyright owners and it is a condition of accessing publications that users recognise and abide by the legal requirements associated with these rights.

\section{Takedown policy}

If you believe that this document breaches copyright please refer to the University of Manchester's Takedown Procedures [http://man.ac.uk/04Y6Bo] or contact uml.scholarlycommunications@manchester.ac.uk providing relevant details, so we can investigate your claim.

\section{OPEN ACCESS}


Draft VERSion November 15, 2018

Typeset using $\mathrm{LAT}_{\mathrm{E}} \mathrm{X}$ preprint2 style in AASTeX62

\section{A SOFIA Survey of [CII] IN the GalaXy M51 I. [C II] AS A TRACER of Star Formation}

Corresponding author: Jorge L. Pineda

Jorge.Pineda@jpl.nasa.gov

Jorge L. Pineda, ${ }^{1}$ Christian Fischer, ${ }^{2}$ Maria Kapala, ${ }^{3}$ Jürgen Stutzki, ${ }^{4}$ Christof Buchbender, ${ }^{4}$ Paul F. Goldsmith, ${ }^{5}$ Monika Ziebart, ${ }^{4}$ Simon C. O. Glover,${ }^{6}$ Ralf S. Klessen, ${ }^{7}$ Jin Koda,${ }^{8}$ Carsten Kramer, ${ }^{9}$ Bhaswati Mookerjea, ${ }^{10}$ Karin Sandstrom,${ }^{11}$ Nick Scoville, ${ }^{12}$ AND Rowan Smith ${ }^{13}$

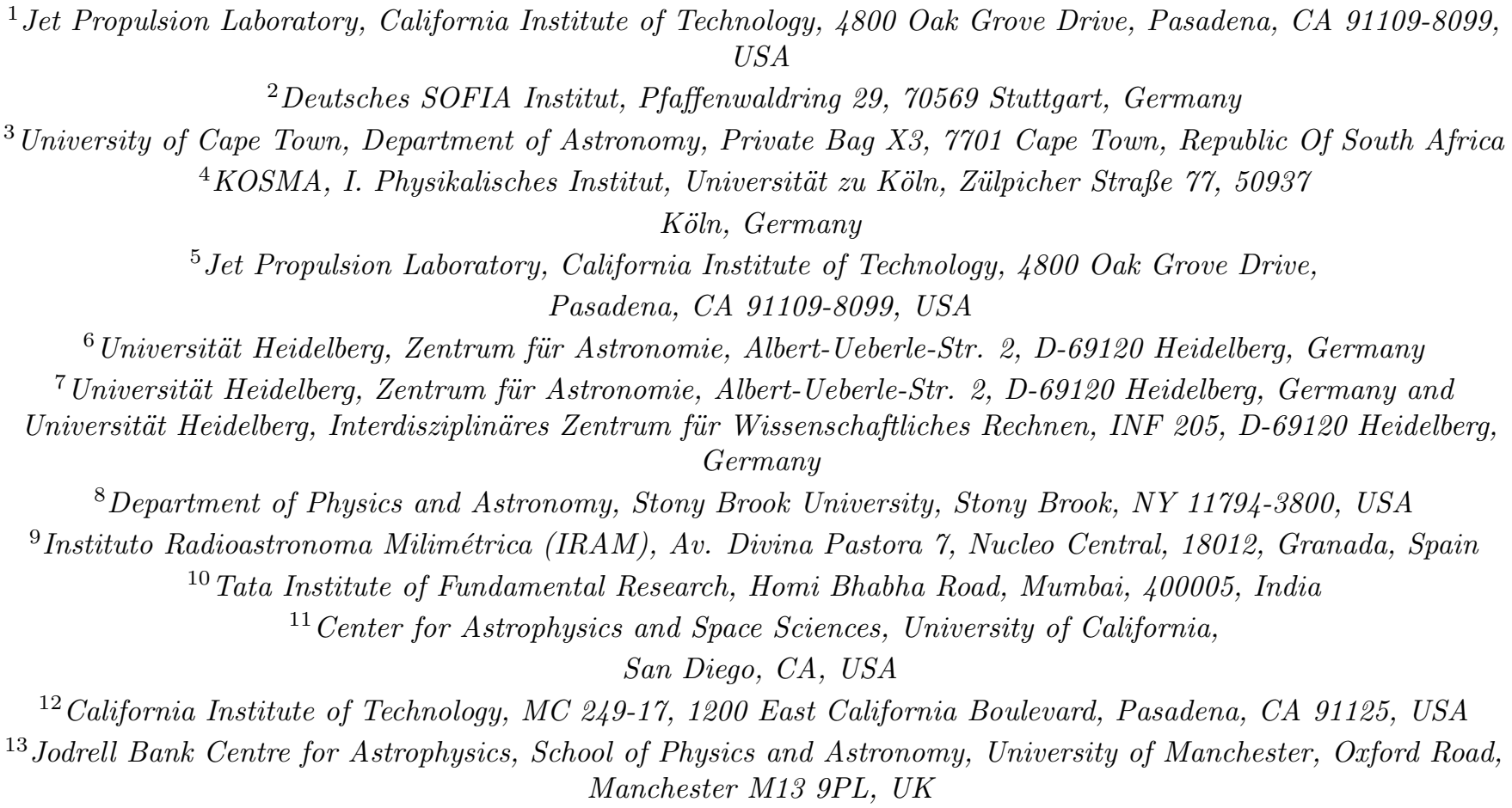
USA

We present a [C II] $158 \mu \mathrm{m}$ map of the entire M51 (including M51b) grand-design spiral galaxy observed with the FIFI-LS instrument on SOFIA. We compare the [C II] emission with the total far-infrared (TIR) intensity and star formation rate (SFR) surface density maps (derived using $\mathrm{H} \alpha$ and $24 \mu \mathrm{m}$ emission) to study the relationship between $[\mathrm{C}$ II] and the star formation activity in a variety of environments within M51 on scales of $16^{\prime \prime}$ corresponding to $\sim 660 \mathrm{pc}$. We find that $[\mathrm{C}$ II] and the SFR surface density are well correlated in the central, spiral arm, and inter-arm regions. The correlation is in good agreement with that found for a larger sample of nearby galaxies at kpc scales. We find that the SFR, and [C II] and TIR luminosities in M51 are dominated by the 

50 of diffuse neutral interstellar gas and therefore 51 plays a critical role in the thermal balance of 52 the interstellar medium of galaxies (Dalgarno \& 53 McCray 1972). Interstellar gas is heated by en54 ergetic electrons that are emitted by dust grains 55 that absorb far-ultraviolet photons from mas56 sive stars (Spitzer 1948). [C II] emission is thus 57 related to the energy input from massive stars 58 to the interstellar medium, making it the bright59 est far-infrared line (Stacey et al. 1991) and an 60 important tracer of the star formation activity ${ }_{61}$ in galaxies. Redshifted [C II] line emission has ${ }_{62}$ been used to study the properties of unresolved 63 galaxies in the early Universe with ALMA and ${ }_{64}$ NOEMA (Carilli \& Walter 2013). It is how${ }_{65}$ ever important to study the relationship be66 tween star formation and the [C II] emission in 67 the local Universe, where different environments ${ }_{68}$ (nuclei, spiral arms, etc.) can be separated, and 69 their relative contribution to the observed star 70 formation tracers can be determined.

${ }_{71}$ The $[\mathrm{C} \mathrm{II}]$ emission and star-formation rate 72 have been observed to be well correlated in the 73 Milky Way (Pineda et al. 2014), within nearby 74 galaxies (Herrera-Camus et al. 2015), in the in75 tegrated emission of unresolved nearby galaxies 76 (de Looze et al. 2011), and even in a sample 77 of "normal" high-redshift galaxies (Capak et al.
78 2015). However, large [C II] intensity deficits 79 (reduced [C II] emission for a given far-infrared 80 (FIR) intensity) have been observed in ultra81 luminous infrared galaxies (e.g. Malhotra et al. 82 2001; Díaz-Santos et al. 2013; Díaz-Santos et al. 83 2017), which has been interpreted as [C II] fail84 ing to trace the (possibly enhanced) star forma85 tion activity in these galaxies. The origin of this 86 deficit remains elusive, with several possibilities 87 being suggested in the literature that might res8 duce the $[\mathrm{C}$ II $]$ intensity and/or increase the FIR 89 intensity (e.g. Graciá-Carpio et al. 2011; Kapala 90 et al. 2017; Díaz-Santos et al. 2013; Goicoechea 91 et al. 2015; Langer \& Pineda 2015; Smith et al. 92 2017). Observations of nearby galaxies provide 93 an important opportunity to study the origin of 94 the [C II] deficit and its relationship to the dif95 ferent environments in these galaxies in detail. 96 In this Letter we present a complete [C II] map 97 of the M51 galaxy observed with the FIFI-LS 98 instrument on SOFIA. This map was obtained 99 as part of a Joint Impact Proposal (program ID 100 04_0116) that also includes a velocity resolved 101 map of M51 obtained with the upGREAT in102 strument on SOFIA. In this Letter, we use the 103 FIFI-LS [C II] map to study the relationship of 104 this spectral line to star formation over the en105 tire disk of the M51, including its companion 106 M51b (NGC5195). The velocity-resolved spec107 tral map of M51 will be presented in a separate 108 paper with emphasis on the gas kinematics. 
M51 is a nearby grand design spiral at a dis110 tance of 8.5 Mpc (McQuinn et al. 2016), with an 111 inclination angle of $24^{\circ}$ (Daigle et al. 2006). It 112 is interacting with a smaller companion galaxy, 113 M51b , classified as a barred lenticular (SB0 114 pec; Sandage \& Tammann 1981) and a LINER 115 galaxy (Ho et al. 1997). Partial maps and in116 dividual positions of M51 have been presented 117 based on observations with the KAO (Nikola 118 et al. 2001) and ISO (Kramer et al. 2005) at 119 resolutions of $55^{\prime \prime}$ and $80^{\prime \prime}$, respectively, which 120 were insufficient to separate different environ121 ments within the galaxy. Parkin et al. (2013) 122 presented [C II] observations at $12^{\prime \prime}$ angular res123 olution in M51 using the Herschel/PACS instru124 ment, but focused only on the inner parts of ${ }_{125}$ M51. With the complete [C II] map of the M51 126 and M51b galaxies we are able to study the re${ }_{127}$ lationship between the [C II] emission and the 128 star formation activity in a wide range of envi129 ronments.

130 This paper is organized as follows. In Sec131 tion 2 we detail the FIFI-LS observations and 132 data reduction. We also describe the ancillary 133 data we use to determine the star-formation 134 rate (SFR) and total far-infrared intensity ${ }_{135}$ (TIR) in M51. In Section 3 we compare the ${ }_{136}$ [C II], TIR intensities, and the SFR surface den137 sity in M51. We discuss the results of this com138 parison in Section 4, and give the conclusions 139 of this work in Section 5. ${ }_{143}$ around $157.8 \mu \mathrm{m}$, with the Far Infrared Field${ }_{144}$ Imaging Line Spectrometer (FIFI-LS) instru145 ment (Colditz et al. 2012; Klein et al. 2014) 146 aboard the Stratospheric Observatory For In147 frared Astronomy (SOFIA; Young et al. 2012). ${ }_{148}$ Details on the FIFI-LS instrument, observing 149 schemes, and atmospheric correction can found 150 in Fischer et al. (2018). Here we present data
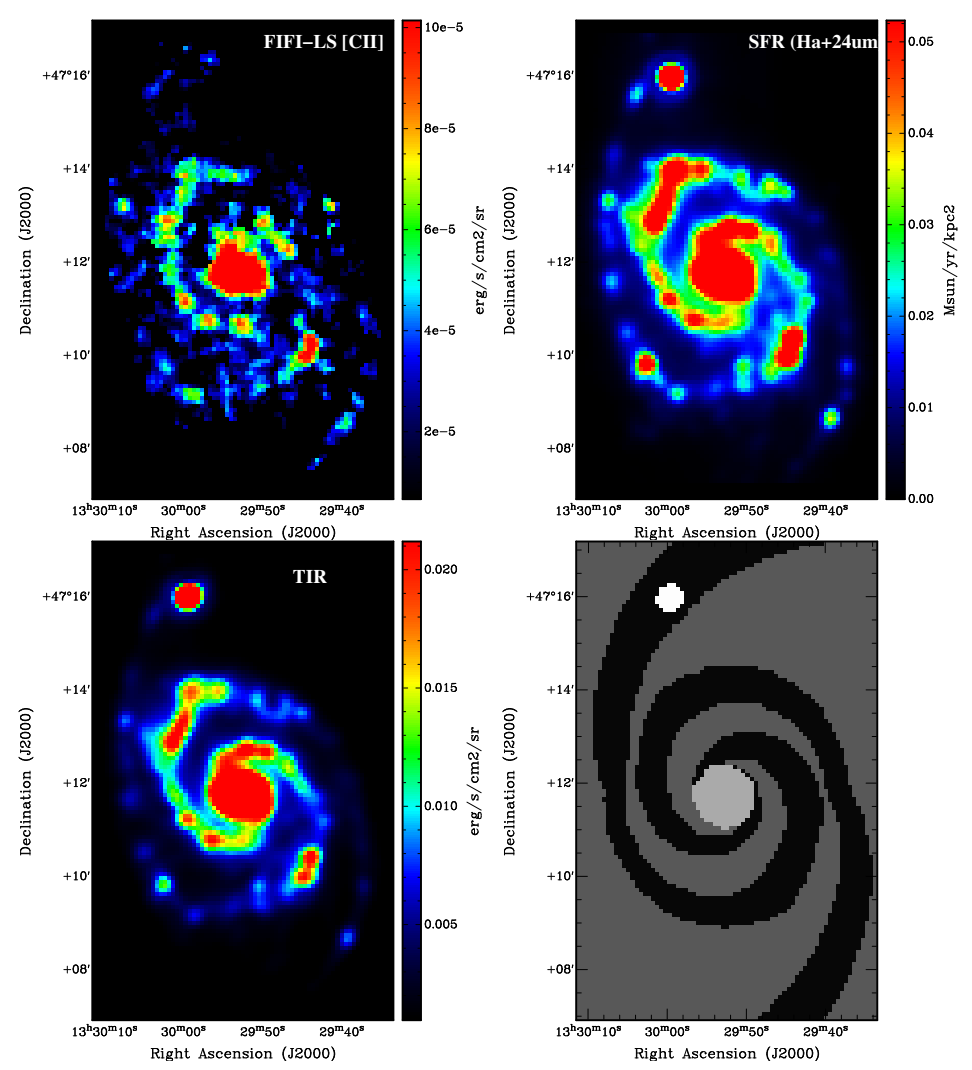

Figure 1. Images of the galaxy M51 in [C II], the star formation rate surface density $\left(\Sigma_{\mathrm{SFR}}\right)$, and the total infrared intensity (TIR). We also include a mask image showing the different environments studied here, center, arms, inter-arms, and M51b, indicated by light-grey, black, dark-grey, and white regions, respectively.

151 taken with the $115-203 \mu \mathrm{m}$ channel that has 152 a $5 \times 5$ pixel footprint on the sky, each with a 153 size of $12^{\prime \prime} \times 12^{\prime \prime}$ yielding a field-of-view of ${ }_{154} 1^{\prime} \times 1^{\prime}$. Each pixel is a so-called "spaxel", which 155 means that internally the light is dispersed spec156 trally (using a grating) over 16 pixels for each 157 spaxel, providing an integral-field data cube 158 covering a total spectral bandwidth between 1591500 and $3000 \mathrm{kms}^{-1}$, with a spectral resolu160 tion of $270 \mathrm{~km} \mathrm{~s}^{-1}$. Our data were acquired in 161 symmetric chop mode with a full throw of $8^{\prime}$. 162 The M51 map is a mosaic of 181 fields of $1^{\prime} \times$ ${ }_{163} 1^{\prime}$ in a grid with $30^{\prime \prime}$ spacing to create half164 pixel sampling and some redundancy in the 165 data set. The on-source integration time per 166 point is $120 \mathrm{~s}$. With the overheads for chop- 
167 ping and telescope motion, the whole map took 168 about $15 \mathrm{~h}$. We assume a diffraction-limited 169 telescope with a point spread function (PSF) 170 having a FWHM of $16^{\prime \prime}(660 \mathrm{pc}$ at a distance of $1718.5 \mathrm{Mpc})$. The data were taken on 11 SOFIA 172 flights in two series between February 2016 and 173 March 2017. Data reduction was carried out us174 ing the FIFI-LS data reduction pipeline (Vacca 175 2016). We fitted a linear baseline to each spec176 trum and integrated the emission within the $177[\mathrm{C} \mathrm{II}]$ spectral region to obtain the integrated 178 intensity map. final [C II] map has a pixel 179 size of $5.3^{\prime \prime}$ and has a typical r.m.s. noise of $1806.6 \times 10^{-6} \mathrm{erg} \mathrm{s}^{-1} \mathrm{~cm}^{-2} \mathrm{sr}^{-1}$.

181 Flux calibration is established by observing 182 standard calibration sources. The absolute am183 plitude calibration uncertainty is assumed to be ${ }_{184} 20 \%$, of which $10 \%$ is the relative uncertainty 185 from FIFI-LS seen between flight series and dif186 ferent calibrators, and the rest is the uncer187 tainty in the atmospheric transmission correc188 tion. We compared the intensities of our FIFI189 LS map in the inner parts of M51 to that ob190 served with Herschel/PACS (Parkin et al. 2013) 191 smoothed to an angular resolution that matched 192 that of FIFI-LS. We found that the FIFI-LS 193 fluxes are systematically lower by a factor of $194 \sim 1.26$ compared with those from PACS. While 195 this factor is within the uncertainties of our ob196 servations, we nevertheless apply this correction 197 factor to the FIFI-LS intensities used in the 198 analysis presented here.

\subsection{TIR and SFR maps}

200

We used Spitzer and Herschel mid and far201 infrared continuum maps (Kennicutt et al. 202 2011) to derive the TIR intensity $\left(I_{\mathrm{TIR}}\right)$ and ${ }_{203}$ SFR surface density $\left(\Sigma_{\mathrm{SFR}}\right)$ in M51. We es204 timated the TIR intensity (i.e. the infrared 205 intensity integrated between 3 and $1100 \mu \mathrm{m}$; in 206 units of erg s${ }^{-1} \mathrm{~cm}^{-2} \mathrm{sr}^{-1}$ ) using,

Table 1. M51 Luminosities

\begin{tabular}{lcccc}
\hline \hline Region & $\begin{array}{c}\text { \# of } \\
\text { pixels }^{\mathrm{a}}\end{array}$ & $\begin{array}{c}L([\mathrm{C} \mathrm{II}]) \\
{[\mathrm{erg} / \mathrm{s}]}\end{array}$ & $\begin{array}{c}L(\mathrm{TIR}) \\
{[\mathrm{erg} / \mathrm{s}]}\end{array}$ & $\begin{array}{c}\text { SFR } \\
{\left[M_{\odot} / \mathrm{yr}\right]}\end{array}$ \\
\hline Center & 182 & $1.2 \times 10^{41}$ & $3.8 \times 10^{43}$ & 0.9 \\
Arms & 2865 & $2.5 \times 10^{41}$ & $9.0 \times 10^{43}$ & 2.5 \\
Inter-arm & 7382 & $8.5 \times 10^{40}$ & $4.7 \times 10^{43}$ & 1.2 \\
M51b & 35 & $7.0 \times 10^{38}$ & $8.4 \times 10^{42}$ & 0.2 \\
\hline Total & 10464 & $4.5 \times 10^{41}$ & $1.8 \times 10^{44}$ & 4.8 \\
\hline
\end{tabular}

$a_{\text {Total number of pixels in mask. }}$

$$
\begin{aligned}
I_{\mathrm{TIR}}=0.95 \nu I_{\nu, 8 \mu \mathrm{m}} & +1.15 \nu I_{\nu, 24 \mu \mathrm{m}} \\
& +\nu I_{\nu, 70 \mu \mathrm{m}}+\nu I_{\nu, 160 \mu \mathrm{m}},
\end{aligned}
$$

where all specific intensities, $I_{\nu}$, are in units of in $\mathrm{erg} \mathrm{s}^{-1} \mathrm{~cm}^{-2} \mathrm{sr}^{-1} \mathrm{~Hz}^{-1}$ (Croxall et al. 2012). The surface density of recent star formation (in units of $\mathrm{M}_{\odot} \mathrm{yr}^{-1} \mathrm{kpc}^{-2}$ ) was estimated using $24 \mu \mathrm{m}$ and $\mathrm{H} \alpha$ maps following Gallagher et al. (2018),

$$
\Sigma_{S F R}=634 I_{\mathrm{H} \alpha}+0.0025 I_{24 \mu m},
$$

207 where the intensity of $I_{\mathrm{H} \alpha}$ is in units of $208 \mathrm{erg} \mathrm{s}^{-1} \mathrm{~cm}^{-2} \mathrm{sr}^{-1}$, and $I_{24 \mu \mathrm{m}}$ in units of $\mathrm{MJy} \mathrm{sr}^{-1}$. ${ }_{209}$ The $\mathrm{H} \alpha$ and $24 \mu \mathrm{m}$ continuum maps were ob210 served as part of the Spitzer/SAGE survey 211 (Kennicutt et al. 2003). Both maps were 212 smoothed with a Gaussian kernel and regrid213 ded to match the resolution and grid of the ${ }_{214}$ FIFI-LS [C II] map. We estimated the rms 215 noise of $I_{\text {TIR }}$ and $\Sigma_{\text {SFR }}$ by calculating the stan216 dard deviation in regions in the maps that are 217 removed from the galaxy. The rms noise is ${ }_{218} 1.3 \times 10^{-3} \mathrm{M}_{\odot} \mathrm{yr}^{-1} \mathrm{kpc}^{-2}$ for the SFR surface 219 density, and $2.5 \times 10^{-4} \mathrm{erg} \mathrm{s}^{-1} \mathrm{~cm}^{-2} \mathrm{sr}^{-1}$ for the 220 TIR intensity.

221

222 In Figure 1 we show the FIFI-LS [C II] map of ${ }_{223}$ M51 together with those of $\Sigma_{\mathrm{SFR}}$ and $I_{\mathrm{TIR}}$. We 

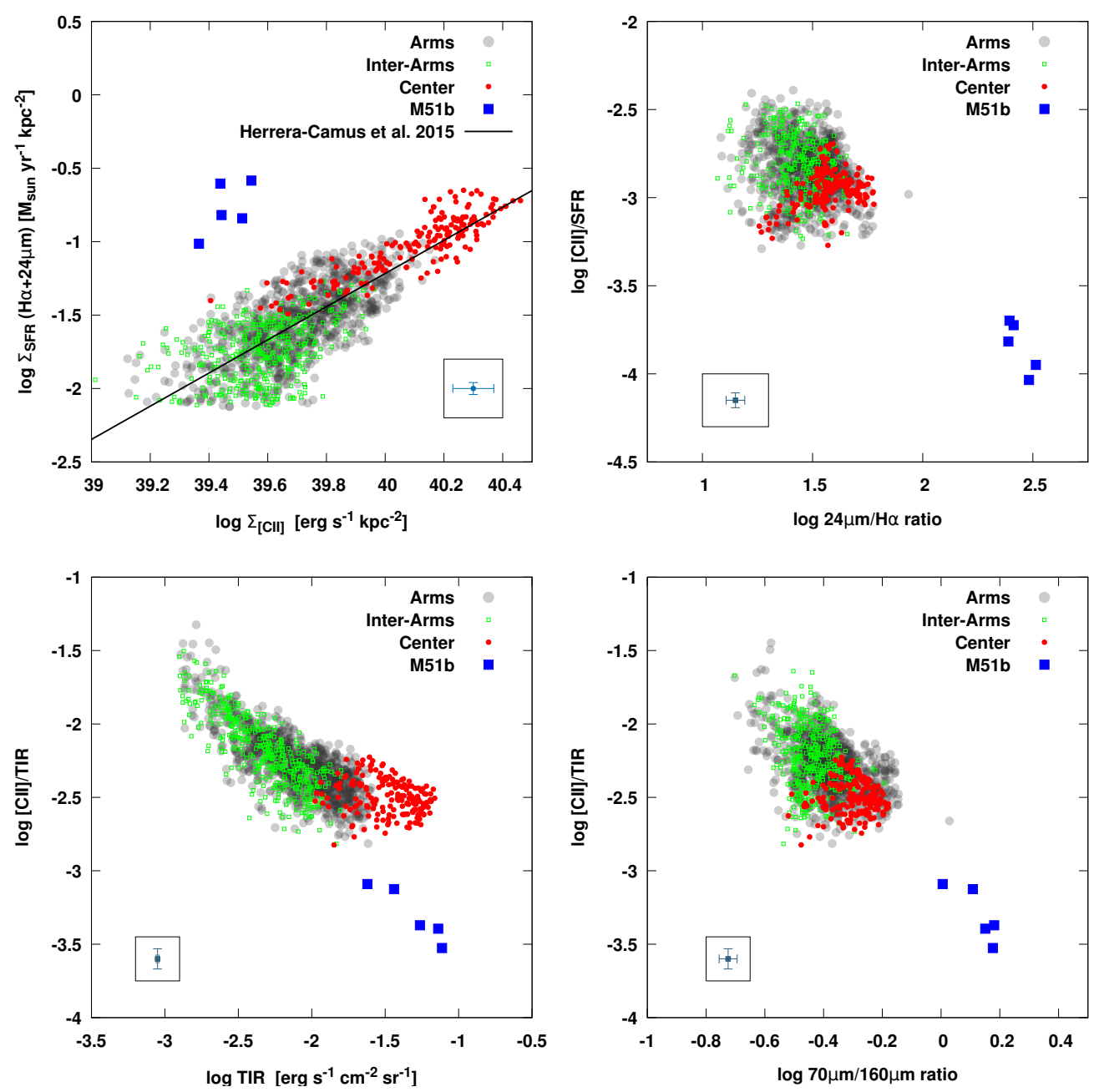

Figure 2. (Upper left panel) The star formation rate surface density as a function of the [C II] luminosity per unit area. The straight line corresponds to the fit of this relationship obtained for a sample of 46 nearby galaxies presented by Herrera-Camus et al. (2015). (Lower left panel) Ratio of [C II] to TIR intensity as a function of the TIR intensity. (Upper right panel) The ratio of the [C II] luminosity per unit area to the star formation rate surface density as a function of the $24 \mu \mathrm{m}$ to $\mathrm{H} \alpha$ ratio. (Lower right panel) Ratio of [C II] to TIR intensity as a function of the $70 \mu \mathrm{m} / 160 \mu \mathrm{m}$ ratio. In all plots different environments defined in Figure 1 are color coded. Typical error bars are shown inside a box. All data points shown are above the $5 \sigma$ level in each quantity.

224 also include a mask that is used to separate dif225 ferent environments in M51. These are the cen226 ter, arms, inter-arms, and the M51b companion 227 galaxy. The arm regions were defined following 228 a geometrical model of the spiral structure in 229 M51 (Pineda et al. 2018, in prep.) and the cen230 ter and M51b masks were defined as pixels with 231 a K-band magnitude (tracing the stellar mass) 232 larger than $17.2 \mathrm{mag}$ and $16 \mathrm{mag}$, respectively, 233 at $16^{\prime \prime}$ resolution. All three images are morpho-
234 logically similar, showing peaks at the center, 235 northern, and southern spiral arms. The only 236 exception is the M51b galaxy that is bright in ${ }_{237}$ the $\Sigma_{\mathrm{SFR}}$ and $I_{\mathrm{TIR}}$ maps but much fainter in the 238 [C II] map.

239 The upper left panel of Figure 2 shows a pixel240 by-pixel comparison between the [C II] luminos${ }_{241}$ ity per unit area observed in M51 and the SFR 242 surface density. Data points from the different ${ }_{243}$ mask regions defined in Figure 1 (arms, inter- 
244 arms, center, and M51b) are color coded. We 245 also show a straight line that corresponds to 246 the $\Sigma_{\text {SFR }}-\Sigma_{[\mathrm{CII}]}$ relationship found by Herrera247 Camus et al. (2015) in a sample of 46 nearby 248 galaxies observed with Herschel/PACS. While 249 the [C II] intensity range of the center, spiral 250 arms, and inter-arm regions in M51 differ, they 251 all show a good correlation between $\Sigma_{\text {SFR }}$ and ${ }_{252} \Sigma_{[\mathrm{CII}]}$. The data points in these regions are also 253 in agreement with the $\Sigma_{\mathrm{SFR}}-\Sigma_{\text {[CII] }}$ relationship 254 of the galaxy sample studied by Herrera-Camus 255 et al. (2015). However, M51b shows signifi256 cantly fainter [C II] emission with respect to the 257 SFR surface density, suggesting a [C II] deficit 258 similar to that seen in ultra-luminous infrared 259 galaxies (ULIRGS). A moderate deficit is also 260 seen in pixels at M51's center.

${ }_{261}$ The lower left panel of Figure 2 shows the ${ }_{262}[\mathrm{C} \mathrm{II}]$ to TIR intensity ratio as a function of 263 the TIR intensity. The data points center, arm, 264 and inter-arm regions are typically in the $10^{-3}$ 265 to $10^{-2}$ range observed in normal galaxies (e.g. 266 Kapala et al. 2015; Kramer et al. 2013). M51b, 267 exhibits much lower values of the $[\mathrm{C} \mathrm{II}] / \mathrm{TIR}$, 268 as low as $\sim 10^{-4}$. Such low values are typical 269 of ULIRGS (Díaz-Santos et al. 2013), but note 270 that the TIR luminosity of M51b (Table 1) is at 271 least two orders of magnitude lower than that 272 of typical ULIRGS.

273 The upper right panel of Figure 2 compares 274 the $\Sigma_{[\mathrm{CII}]} / \Sigma_{\mathrm{SFR}}$ ratio to the $24 \mu \mathrm{m} / \mathrm{H} \alpha$ ratio 275 to study whether there are systematic differ276 ences between star forming regions in the arms 277 and more diffuse regions in the inter-arms of 278 M51. We find no significant difference between 279 the $24 \mu \mathrm{m} / \mathrm{H} \alpha$ in the arm (average ratio 31.5 ) 280 and inter-arm (average ratio 27.5) regions of 281 M51. The central region has a somewhat larger $28224 \mu \mathrm{m} / \mathrm{H} \alpha$ ratio of 39 . However, M51b devi283 ates significantly with an average $24 \mu \mathrm{m} / \mathrm{H} \alpha$ ra284 tio of 276. This deviation is a result of en285 hanced $24 \mu \mathrm{m}$ emission rather than fainter $\mathrm{H} \alpha$ 286 emission. We show in the lower right panel of

287 Figure 2, the $[\mathrm{C} \mathrm{II}] /$ TIR ratio as a function of 288 the $70 \mu \mathrm{m} / 160 \mu \mathrm{m}$ ratio, which is a proxy for the 289 dust temperature. The arm and inter-arm re290 gions have similar average $70 \mu \mathrm{m} / 160 \mu \mathrm{m}$ ratio 291 (0.35 and 0.4, respectively) while the central re292 gion has a slightly larger average value (0.5). 293 M51b shows an enhanced average $70 \mu \mathrm{m} / 160 \mu \mathrm{m}$ 294 ratio of 1.3 , which indicates that the reduced $295[\mathrm{C} \mathrm{II}] /$ TIR ratio in this galaxy is related to an 296 increase in dust temperature. The suggested 297 enhancement of the dust temperature in M51b 298 is consistent with that estimated by Mentuch ${ }_{299}$ Cooper et al. (2012). A similar dependence of 300 the $[\mathrm{C}$ II $] /$ TIR ratio to dust temperature seen in 301 Figure 2 has been observed in a larger sample 302 of galaxies (including ULIRGS; Herrera-Camus 303 et al. 2015; Díaz-Santos et al. 2017; Díaz-Santos 304 et al. 2013; Lu et al. 2015).

305 311 luminosities in M51 are dominated by the disk 312 of M51 (arm and inter-arm regions) represent313 ing about $75 \%$ of the total value of these quan314 tities, even though the central region has the 315 highest surface density. The larger luminosity 316 in the disk of M51 is a result of these quanti317 ties being extended over a larger area than that 318 of the central and M51b regions. This suggests 319 that if M51 were at a greater distance, and thus 320 were unresolved, the measured SFR and [C II] 321 and TIR luminosities would be dominated by 322 the galaxy's disk rather than by its central re323 gion.

324 4.2. The origin of the $[C \mathrm{II}]$ deficit in $M 51 b$ 325 In the upper panel of Figure 3 we show [C II] 326 emission from M51b in the form of contours. 327 We also show the TIR emission. While most 328 of the TIR emission (and inferred SFR) peaks 

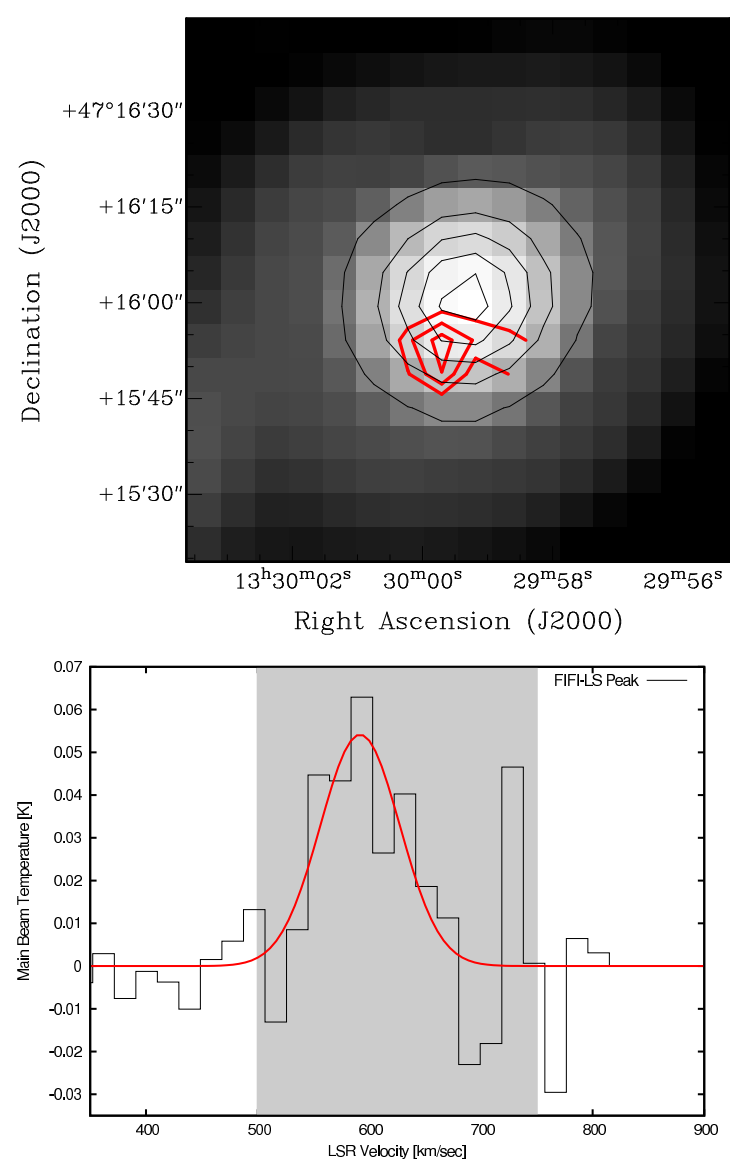

Figure 3. (Upper panel) Image of M51b observed in [C II] with SOFIA/FIFI-LS. The red contours correspond to $65 \%$ of the peak $\left(2.9 \times 10^{-5}\right.$ $\left.\mathrm{erg} \mathrm{s}^{-1} \mathrm{~cm}^{-2} \mathrm{sr}^{-1}\right)$ to $100 \%$ in steps of $15 \%$. The grey-scale and black contours indicate the TIR intensity. (Lower panel) [CII] spectrum observed with the SOFIA/upGREAT instrument resulting from the average of the spectra above the $2.0 \times 10^{-5}$ $\mathrm{erg} \mathrm{s}^{-1} \mathrm{~cm}^{-2} \mathrm{sr}^{-1} 3 \sigma$ level in the FIFI-LS image and $\mathrm{S}-\mathrm{W}$ from M51b's center. The red line denotes the result of a Gaussian fit and the grey area the expected velocity range of the integrated $\mathrm{CO}$ emission observed in M51b (Kohno et al. 2002).

329 at the center of the galaxy, [C II] shows emis330 sion only from the South-West $(\mathrm{S}-\mathrm{W})$ side of 331 the TIR peak. CO observations show a molec332 ular disk in M51b that extends to the $\mathrm{S}-\mathrm{W}$ and 333 North-East (N-E) from its center (Kohno et al. 334 2002; Alatalo et al. 2016). The $\mathrm{S}-\mathrm{W}$ and $\mathrm{N}-\mathrm{E}$ 335 components have $\mathrm{CO}$ velocities of $\sim 580 \mathrm{~km} \mathrm{~s}^{-1}$
336 and $\sim 680 \mathrm{~km} \mathrm{~s}^{-1}$, respectively (Kohno et al. 337 2002). We also observed this region with the up338 GREAT instrument with velocity resolved ob339 servations but could not detect [C II] in indi340 vidual pixels. However, averaging the spectra 341 above the $3 \sigma$ contour in the FIFI-LS map and ${ }_{342} \mathrm{~S}-\mathrm{W}$ from M51b's center results in the spec343 trum shown in the lower panel of Figure 3. The 344 [C II] line is clearly detected at the $\mathrm{S}-\mathrm{W}$ with 345 a peak at $580 \mathrm{~km} \mathrm{~s}^{-1}$, which is consistent with 346 the $\mathrm{CO}$ velocity in this region. The total in347 tegrated intensity of the observed spectrum is $3482.4 \times 10^{-5} \mathrm{erg} \mathrm{s}^{-1} \mathrm{~cm}^{-2} \mathrm{sr}^{-1}$, which is consistent 349 with the average intensity of the FIFI-LS map 350 in this area of $2.0 \times 10^{-5} \mathrm{erg} \mathrm{s}^{-1} \mathrm{~cm}^{-2} \mathrm{sr}^{-1}$. We 351 also averaged the upGREAT spectra in the $\mathrm{N}-$ $352 \mathrm{E}$ part of the galaxy, but could not detect any 353 emission.

354 M51b is intrinsically very faint in [C II] con355 sidering the observed TIR intensity and the in356 ferred SFR. Additionally, this galaxy shows en357 hanced $24 \mu \mathrm{m}$ emission (Figure 2) and elevated 358 dust temperature (Mentuch Cooper et al. 2012) 359 compared to M51. M51b is a barred lenticu360 lar galaxy in a post-starburst phase, in which 361 the stellar population is dominated by old stars 362 ( $\gtrsim 10 \mathrm{Gyr}$ ) and massive star-formation is sup363 pressed (Kohno et al. 2002; Alatalo et al. 2016). 364 The lack of massive star formation in M51b 365 is consistent with the faint [C II] emission de366 tected but it is inconsistent with the large TIR 367 emission observed in this galaxy and the high ${ }_{368}$ SFR inferred from $\mathrm{H} \alpha$ and $24 \mu \mathrm{m}$ dust contin369 uum emission.

M51b shows Active Galactic Nuclei (AGN) 371 activity, based on its near-infrared line emis372 sion (Goulding \& Alexander 2009) and there is 373 evidence of AGN feedback producing arcs and 374 shocks in $\mathrm{X}$-ray and $\mathrm{H} \alpha$ emission (Schlegel et al. 375 2016; Rampadarath et al. 2018). X-rays can 376 heat the dust to higher temperatures than FUV 377 emission, and can result in bright infrared emis378 sion, resulting in enhanced values of the TIR 
379 intensity and anomalously large inferred SFR 380 (Voit 1991). Note, however, that the X-ray lu381 minosity in $\operatorname{M} 51 \mathrm{~b}\left(L_{\mathrm{X}}=5.4 \times 10^{38} \mathrm{erg} \mathrm{s}^{-1}\right)$ is low 382 compared to what is typical for galaxies (Ebrero 383 et al. 2009), and is a factor of 30 lower than that 384 at the center of M51 (Brightman et al. 2018). 385 Thus, the AGN in M51b can heat the dust only 386 in its immediate vicinity. Higher resolution im387 ages of the $24 \mu \mathrm{m}$ and $\mathrm{H} \alpha$ emission in $\mathrm{M} 51 \mathrm{~b}$ 388 show a point source at its center that is unre389 solved in the $6^{\prime \prime}(247 \mathrm{pc})$ and $0.31^{\prime \prime}(12 \mathrm{pc})$ reso390 lution of these images, respectively. As the [C II] 391 peak is about $10^{\prime \prime}(\sim 450 \mathrm{pc})$ from the center of ${ }_{392} \mathrm{M} 51 \mathrm{~b}$, it is therefore possible that the bright ${ }_{393}$ fir- and mid-infrared and [C II] emission arises 394 from different locations within the $16^{\prime \prime}$ beam 395 of our observations, with the hot dust associ396 ated to the AGN dominating the mid- and far397 infrared emission and a higher column density, 398 colder dust temperature region contributing to 399 the $[\mathrm{CII}]$ emission. This [C II]-emitting cloud 400 is too far from the AGN to be influenced by 401 its X-ray emission. The evolved stellar popu${ }_{402}$ lation in M51b can also heat the dust but does 403 not contribute significantly to the FUV emis404 sion that heats the gas via photoelectric effect ${ }_{405}$ (Bendo et al. 2012; Kapala et al. 2017). Note, 406 however, that the stellar surface density at the 407 center of M51 is similar to that in M51b, but 408 this region shows only a moderate deficit of [C II] 409 with respect to the TIR emission and inferred ${ }_{410}$ SFR. Thus, an enhanced stellar density is likely 411 not the only mechanism responsible for the low ${ }_{412}[\mathrm{C} \mathrm{II}] / \mathrm{SFR}$ and $[\mathrm{C} \mathrm{II}] /$ TIR ratios in M51b.

\section{$413 \quad 4.3 . \quad[C \mathrm{II}]$ emission at the center of M51}

${ }_{414}$ As shown in Figure 2, there are a number of ${ }_{415}$ pixels at the center of M51 that show a mod416 erate [C II] deficit for a given SFR surface den417 sity. However, this effect is not as pronounced ${ }_{418}$ when $[\mathrm{CII}]$ is compared with the TIR inten${ }_{419}$ sity. In Figure 4 we show the [C II] emission 420 in M51's central regions with contours of the ${ }_{421}$ TIR intensity and inferred SFR. The SFR sur-
422 face density image shows a peak of emission ${ }_{423}$ at its center that is not present in the [C II] 424 image (both the $\mathrm{H} \alpha$ and $24 \mu \mathrm{m}$ show a point425 source emission at this location ). This peak 426 corresponds to the location of the Compton${ }_{427}$ thick AGN activity present in this region (Stauf${ }_{428}$ fer 1982; Fukazawa et al. 2001). Therefore, 429 this region also represents an example of AGN430 powered $\mathrm{H} \alpha$ and $24 \mu \mathrm{m}$ emission, with fainter ${ }_{431}[\mathrm{C}$ II] emission. The peak at the AGN location ${ }_{432}$ can also be seen in the far-infrared images used 433 to estimate the TIR intensity but it is not as ${ }_{434}$ pronounced as for the shorter wavelength data 435 used to determine the SFR surface density.

\section{CONCLUSIONS}

${ }_{437}$ We presented a [C II] emission map over the ${ }_{438}$ entire M51 and M51b galaxies observed with 439 the SOFIA/FIFI-LS instrument. We compared 440 the [C II] emission with the total far-infrared ${ }_{441}$ intensity and star formation rate surface den442 sity maps of M51 within a variety of environ${ }_{443}$ ments. We found that [C II] and the SFR sur${ }_{444}$ face density are well correlated in the central, 445 spiral arm, and inter-arm regions. The correla446 tion is in good agreement with that found for a 447 larger sample of nearby galaxies at kpc scales. ${ }_{448}$ We found that the SFR and [C II] and TIR lumi${ }_{449}$ nosities in M51 are dominated by the extended 450 emission in M51's disk. The companion galaxy ${ }_{451}$ M51b, however, shows a deficit of [C II] emis452 sion compared with the TIR emission and in453 ferred SFR surface density, with [C II] emission ${ }_{454}$ detected only in the $\mathrm{S}-\mathrm{W}$ part of this galaxy. ${ }_{455}$ We find that this [C II] deficit is related to an 456 enhanced dust temperature in this galaxy. We ${ }_{457}$ interpret the faint [C II] emission in M51b to 458 be a result of suppressed star formation in this 459 galaxy, while the bright mid- and far-infrared 460 emission, which drive the TIR and SFR values, 461 are powered by other mechanisms. A similar 462 but less pronounced effect is seen at the loca${ }_{463}$ tion of the black hole in M51's center. The ob464 served [C II] deficit in M51b suggests that this 

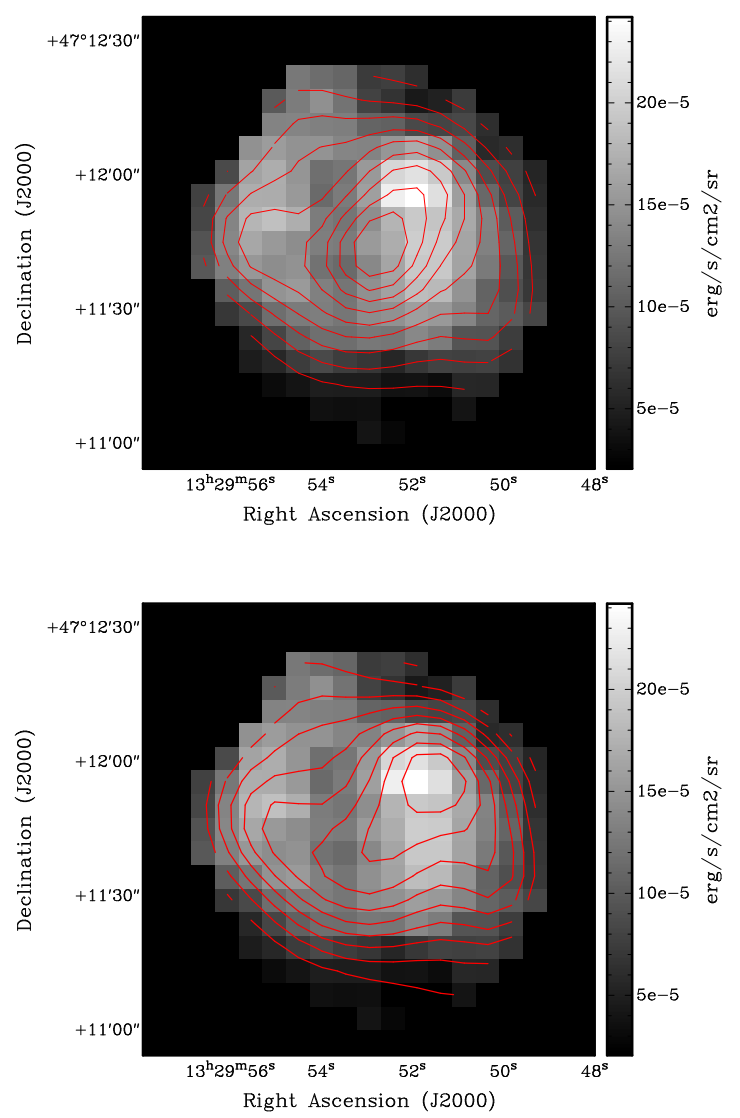

Figure 4. (Upper panel) Image of M51 center observed in [C II] with SOFIA/FIFI-LS overlaid with contours of the inferred SFR surface density. The contour lines range from $10 \%$ of the peak $\left(0.22 \mathrm{M}_{\odot} \mathrm{yr}^{-1}\right)$ to $90 \%$ in steps of $10 \%$. (Lower panel) [C II] image of M51's center overlaid with contours of the TIR surface density. The contour lines correspond range from $10 \%$ of the peak $(0.07$ $\mathrm{erg} \mathrm{s}^{-1} \mathrm{~cm}^{-2} \mathrm{sr}^{-1}$ ) to $90 \%$ in steps of $10 \%$.
465 galaxy represents a valuable laboratory in which 466 to study the origin of the apparent [C II] deficit 467 observed in ultra-luminous infrared galaxies.
${ }_{468}$ Based on observations made with the NASA/DLR 469 Stratospheric Observatory for Infrared Astron470 omy (SOFIA). SOFIA is jointly operated by the 471 Universities Space Research Association, Inc. 472 (USRA), under NASA contract NAS2-97001, 473 and the Deutsches SOFIA Institut (DSI) under ${ }_{474}$ DLR contract 50 OK 0901 to the University of 475 Stuttgart. We thank the staff of the SOFIA 476 Science Center for their help. We also thank 477 an anonymous referee for a number of use478 ful comments that significantly improved the 479 manuscript. Part of the research was carried 480 out at the Jet Propulsion Laboratory, Califor481 nia Institute of Technology, under a contract ${ }_{482}$ with the National Aeronautics and Space Ad483 ministration. (C) 2018. All rights reserved. U.S. ${ }_{484}$ Government sponsorship acknowledged.

\section{REFERENCES}

486 Alatalo, K., Aladro, R., Nyland, K., et al. 2016,

${ }_{487}$ ApJ, 830, 137

488 Bendo, G. J., Boselli, A., Dariush, A., et al. 2012,

$489 \quad$ MNRAS, 419, 1833

490 Brightman, M., Baloković, M., Koss, M., et al. 491 2018, ArXiv e-prints, arXiv:1805.12140

492 Capak, P. L., Carilli, C., Jones, G., et al. 2015,

$493 \quad$ Nature, 522, 455
494 Carilli, C. L., \& Walter, F. 2013, ARA\&A, 51, 105

${ }_{495}$ Colditz, S., Fumi, F., Geis, N., et al. 2012, in

496 Proc. SPIE, Vol. 8446, Ground-based and

497 Airborne Instrumentation for Astronomy IV,

$498 \quad 844617$

499 Croxall, K. V., Smith, J. D., Wolfire, M. G., et al. $500 \quad 2012$, ApJ, 747, 81 
501 Daigle, O., Carignan, C., Amram, P., et al. 2006,

$502 \quad$ MNRAS, 367, 469

503 Dalgarno, A., \& McCray, R. A. 1972, ARA\&A,

$504 \quad 10,375$

505 de Looze, I., Baes, M., Bendo, G. J., Cortese, L.,

506 \& Fritz, J. 2011, MNRAS, 416, 2712

507 Díaz-Santos, T., Armus, L., Charmandaris, V.,

508 et al. 2013, ApJ, 774, 68

509 Díaz-Santos, T., Armus, L., Charmandaris, V.,

$510 \quad$ et al. 2017, ApJ, 846, 32

511 Ebrero, J., Carrera, F. J., Page, M. J., et al. 2009,

$512 \quad$ A\&A, 493, 55

513 Fischer, C., Beckmann, S., Bryant, A., et al. 2018,

514 Journal of Astronomical Instrumentation,

$515 \quad 1840003$

516 Fukazawa, Y., Iyomoto, N., Kubota, A.,

517 Matsumoto, Y., \& Makishima, K. 2001, A\&A,

$518 \quad 374,73$

${ }_{519}$ Gallagher, M. J., Leroy, A. K., Bigiel, F., et al.

$520 \quad 2018$, ApJ, 858, 90

${ }_{521}$ Goicoechea, J. R., Teyssier, D., Etxaluze, M.,

522 et al. 2015, ApJ, 812, 75

523 Goulding, A. D., \& Alexander, D. M. 2009,

524 MNRAS, 398, 1165

${ }_{525}$ Graciá-Carpio, J., Sturm, E., Hailey-Dunsheath,

$526 \quad$ S., et al. 2011, ApJL, 728, L7

${ }_{527}$ Herrera-Camus, R., Bolatto, A. D., Wolfire,

${ }_{528}$ M. G., et al. 2015, ApJ, 800, 1

${ }_{529}$ Ho, L. C., Filippenko, A. V., \& Sargent, W. L. W.

$530 \quad$ 1997, ApJS, 112, 315

${ }_{531}$ Kapala, M. J., Sandstrom, K., Groves, B., et al.

$532 \quad 2015$, ApJ, 798, 24

533 Kapala, M. J., Groves, B., Sandstrom, K., et al.

$534 \quad 2017$, ApJ, 842, 128

535 Kennicutt, R. C., Calzetti, D., Aniano, G., et al.

$536 \quad$ 2011, PASP, 123, 1347

537 Kennicutt, Jr., R. C., Armus, L., Bendo, G., et al.

538 2003, PASP, 115, 928

539 Klein, R., Beckmann, S., Bryant, A., et al. 2014,

540 in Proc. SPIE, Vol. 9147, Ground-based and

${ }_{541}$ Airborne Instrumentation for Astronomy V,

$54291472 \mathrm{X}$
543 Kohno, K., Tosaki, T., Matsushita, S., et al. 2002, $544 \quad$ PASJ, 54, 541

545 Kramer, C., Mookerjea, B., Bayet, E., et al. 2005, ${ }_{546} \quad$ A\&A, 441, 961

547 Kramer, C., Abreu-Vicente, J., García-Burillo, S., 548 et al. 2013, A\&A, 553, A114

549 Langer, W. D., \& Pineda, J. L. 2015, A\&A, 580, $550 \quad$ A5

551 Lu, N., Zhao, Y., Xu, C. K., et al. 2015, ApJL, $552 \quad 802, \mathrm{~L} 11$

553 Malhotra, S., Kaufman, M. J., Hollenbach, D., 554 et al. 2001, ApJ, 561, 766

555 McQuinn, K. B. W., Skillman, E. D., Dolphin, 556 A. E., Berg, D., \& Kennicutt, R. 2016, ApJ, $557 \quad 826,21$

558 Mentuch Cooper, E., Wilson, C. D., Foyle, K.,

559 et al. 2012, ApJ, 755, 165

560 Nikola, T., Geis, N., Herrmann, F., et al. 2001,

561 ApJ, 561, 203

562 Parkin, T. J., Wilson, C. D., Schirm, M. R. P.,

563 et al. 2013, ApJ, 776, 65

564 Pineda, J. L., Langer, W. D., \& Goldsmith, P. F.

565 2014, A\&A, 570, A121

566 Rampadarath, H., Soria, R., Urquhart, R., et al.

567 2018, MNRAS, 476, 2876

568 Sandage, A., \& Tammann, G. A. 1981, A Revised

569 Shapley-Ames Catalog of Bright Galaxies

570 Schlegel, E. M., Jones, C., Machacek, M., \& Vega, L. D. 2016, ApJ, 823, 75

572 Smith, J. D. T., Croxall, K., Draine, B., et al.

573 2017, ApJ, 834, 5

574 Spitzer, Jr., L. 1948, ApJ, 107, 6

575 Stacey, G. J., Geis, N., Genzel, R., et al. 1991,

576 ApJ, 373, 423

577 Stauffer, J. R. 1982, ApJ, 262, 66

578 Vacca, W. 2016, Guest Investigator Handbook for

579 FIFI-LS Data Products, Tech. rep., SOFIA

$580 \quad$ Science Center

581 Voit, G. M. 1991, ApJ, 379, 122

582 Young, E. T., Becklin, E. E., Marcum, P. M.,

583 et al. 2012, ApJL, 749, L17 